\title{
Development of an Intellectual Educational and Diagnostic Complex for the Histological Analysis of Thyroid Tumors
}

\section{Nikitaev V.G. ${ }^{1}$, Pronichev A.N. ${ }^{1}$, Selchuk V. Yu. ${ }^{1,2}$, Druzhinina E.A. ${ }^{1}$, Tavrina N.S. ${ }^{1}$, Dmitrieva V.V. ${ }^{1}$, and Polyakov E.V. ${ }^{1}$}

${ }^{1}$ National Research Nuclear University MEPhl (Moscow Engineering Physics Institute), Kashirskoe shosse 31, 115409, Moscow, Russia

${ }^{2}$ N.N. Blokhin Russian Cancer Research Center, Kashirskoe shosse 23, 115478, Moscow, Russia

\section{Abstract}

The purpose of this project is the creation of an interactive intellectual training system based on the DBMS, which allows to organize and store information, transfer and copy data for employees. The already existing images and their characteristics are structured, a decision support system is developed for the recognition of tumors and

Corresponding Author:

V. Nikitaev

VGNikitayev@mephi.ru

Received: 17 January 2018

Accepted: 25 March 2018

Published: 17 April 2018

Publishing services provided by

Knowledge E

(c) Nikitaev V.G. et al. This article is distributed under the terms of the Creative Commons

Attribution License, which permits unrestricted use and redistribution provided that the original author and source are credited.

Selection and Peer-review under the responsibility of the PhysBioSymp17 Conference Committee. suspicious cells, which saves time for an already experienced oncologist. This system allows to increase the accuracy of diagnosis. Educational and diagnostic complexes help to accumulate experience for young specialists in the field of image analysis, carry out comparative research, which speeds up the learning process, promotes professional development of the specialist.

Keywords: diagnostic,thyroid, oncological, aspiration biopsy.

\section{Introduction}

The death rate from oncological diseases is steadily growing. The solution of this problem directly depends on the timely diagnosis. Early detection of problems and predispositions to them makes a significant contribution to solving the problem of treating thyroid diseases. Risk factors for cancer development can be divided into 4 groups. An example of these factors for the Russian Federation:

- Genetic factors - $15-20 \%$

- The state of the environment - $20-25 \%$

- Medical care - 10-15\%

- Conditions and way of life of people - $50-55 \%$ 
Each of these groups is highly variable, which is an extensive area for research. Such statistics reveal factors that make predictions about threats to human health. Specialists will generate such statistics at different levels of the patient's examination in order to make the diagnosis more accurate.The relevance of the work is to systematize and compare already existing knowledge about thyroid with new data, which makes it possible to detect regularities in the signs, both benign neoplasms, and in developing malignant tumors [1-11].

\section{Materials and Methods}

The structural diagram of the project can be seen in Figure 1.

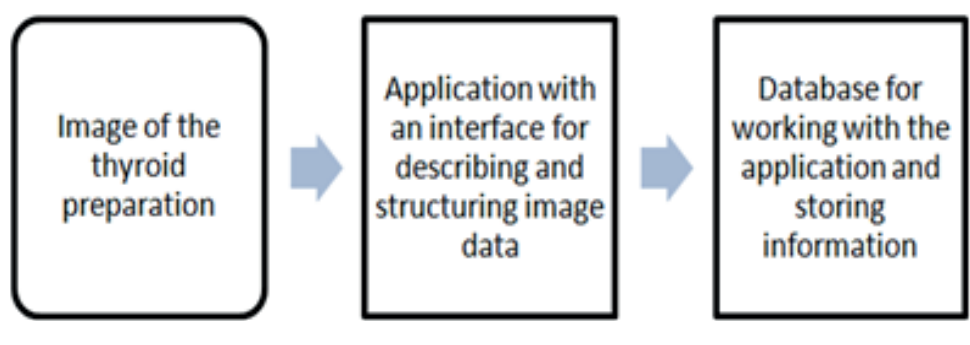

An the output: application for comparison, study of patterns, of search and query generation + database

Figure 1: Structure of the project.

A histological preparation (material) for determining the quality of a thyroid tumor is taken by performing a fine needle aspiration biopsy. With a planned biopsy tissue samples are filled with paraffin, and after hardening, cut into thin plates, and then subjected to staining.

Accelerated method is mainly practiced during the operation, when it is necessary to take an urgent decision on further actions.

In this case, the material is frozen, as well cut into the thinnest plates and studied under a microscope.

The application is based on a specially developed database developed specifically. The main blank table, the columns of which correspond to small filled labels. You can make entries to the main table by selecting an option from the small completed

The object environment is the image of a microscopic preparation of the thyroid gland. They will be described by the user, and we will give him the opportunity to select a description from the already developed list (it is similar to filling in the questionnaire). The classification table (methods) of the preparation developed in conjunction with the doctors of the Federal State Budgetary Institution "National Medical Research Center of Oncology. N.N. Blokhin "of the Ministry of Health of Russia [1-5]

Conceptual model of this complex. This is intended: 
1. For experienced specialists in the field of thyroid diseases. This will allow to structure existing data about patients, make them mobile, useful for future diagnosis and treatment. The doctor will be able to use this system as a navigator for a large amount of data, among which he can quickly find out similar characteristics of preparation. The data can be conveniently stored and transferred to colleagues.

2. The complex is useful for upgrading the skills of young professionals. An experienced oncologist will be able to save time, giving young specialists an opportunity to compare the preparation? Which they study with other preparation in the database. Compare can be visually simultaneously with all information about the image.

\section{Work with application}

Numbered button- to go to additional form-windows, which help you fill the lines of the main table or search in the table.

-There are ordinary elements of the basic form

And elements used during the search for the base, provided the flag is checked (Figure 2)

\section{Results}

Practical application of intelligent educational and diagnostic complex is in medicine. This complex allows building a knowledge base in which the images of the microscopic analysis of thyroid preparations and their description are systematized in accordance with the classification table of the preparation.

Complex is based on a database developed with the help of MYSQL. It is suitable for further development and can use both locally on a computer that is supported MySQL-database and remotely, you need an Internet connection and then you do not need to install the program MYSQL. The whole process is visualized. The developed complex can be useful in describing the symptoms and in the formation of a diagnosis and treatment plan for thyroid tumors (Figure 3) [6, 10-11]. 


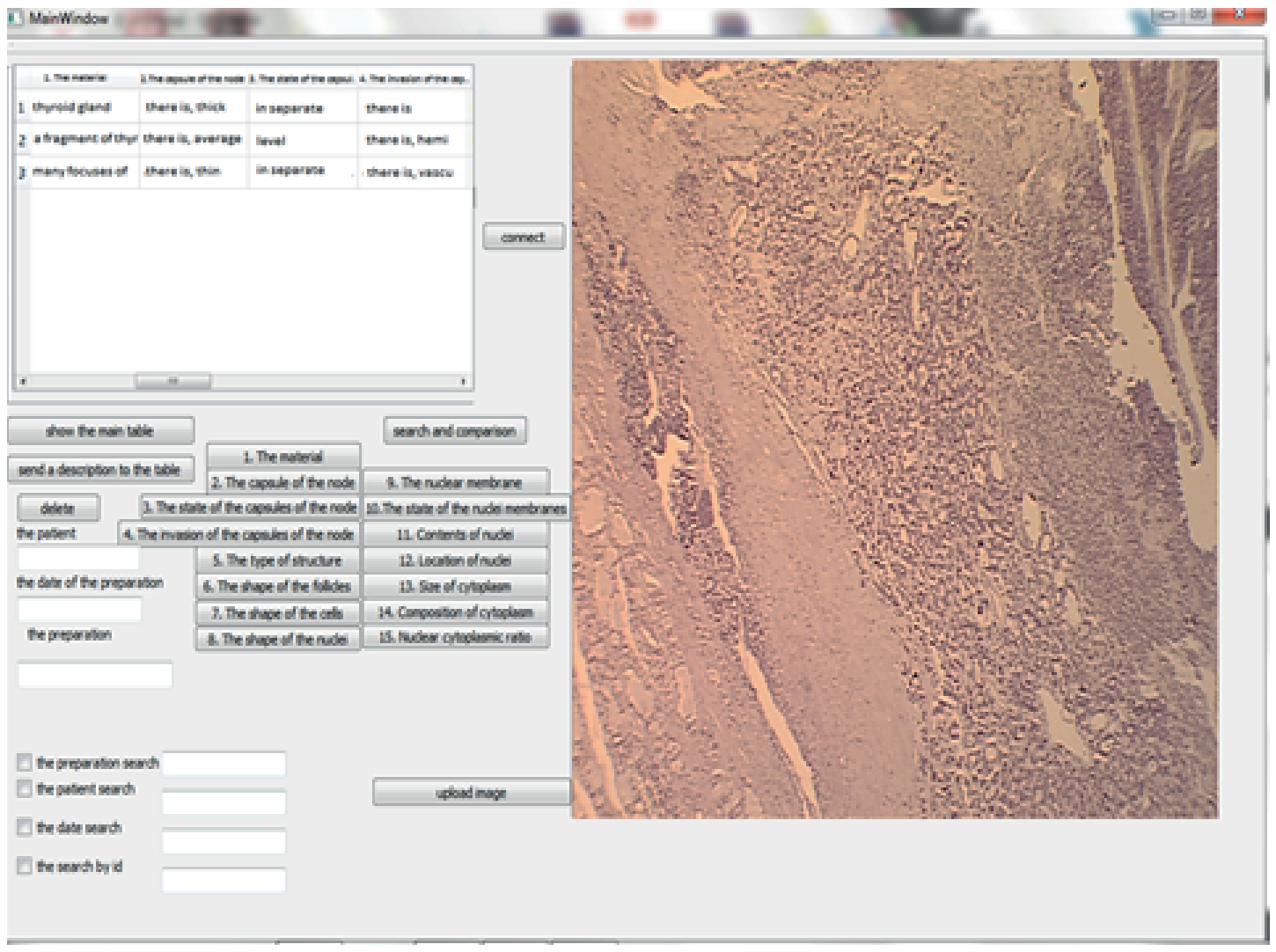

Figure 2: Implementation of the interface to fill the database (the method of filling is similar to filling out the questionnaire - choose the appropriate option from the proposed tables, but you can also enter something in your special field EXTRA.

\section{Conclusion}

The proposed complex is based on the DBMS. This allows you to organize and store information, it's more convenient than a paper image description, it's easy to transfer and copy data to employees, which reduces the risk of loss or confusion.

Multimedia educational and diagnostic complexes help to conduct research, accumulate experience for young specialists in the field of recognition, image processing. This complex accelerates the learning process and saves time for the specialist pathologist and trained specialist.

\section{Acknowledgement}

This work was supported by the MEPhl Academic Excellence Project (agreement with the Ministry of Education and Science of the Russian Federation of August 27, 2013, project no. 02.a03.21.0005). 


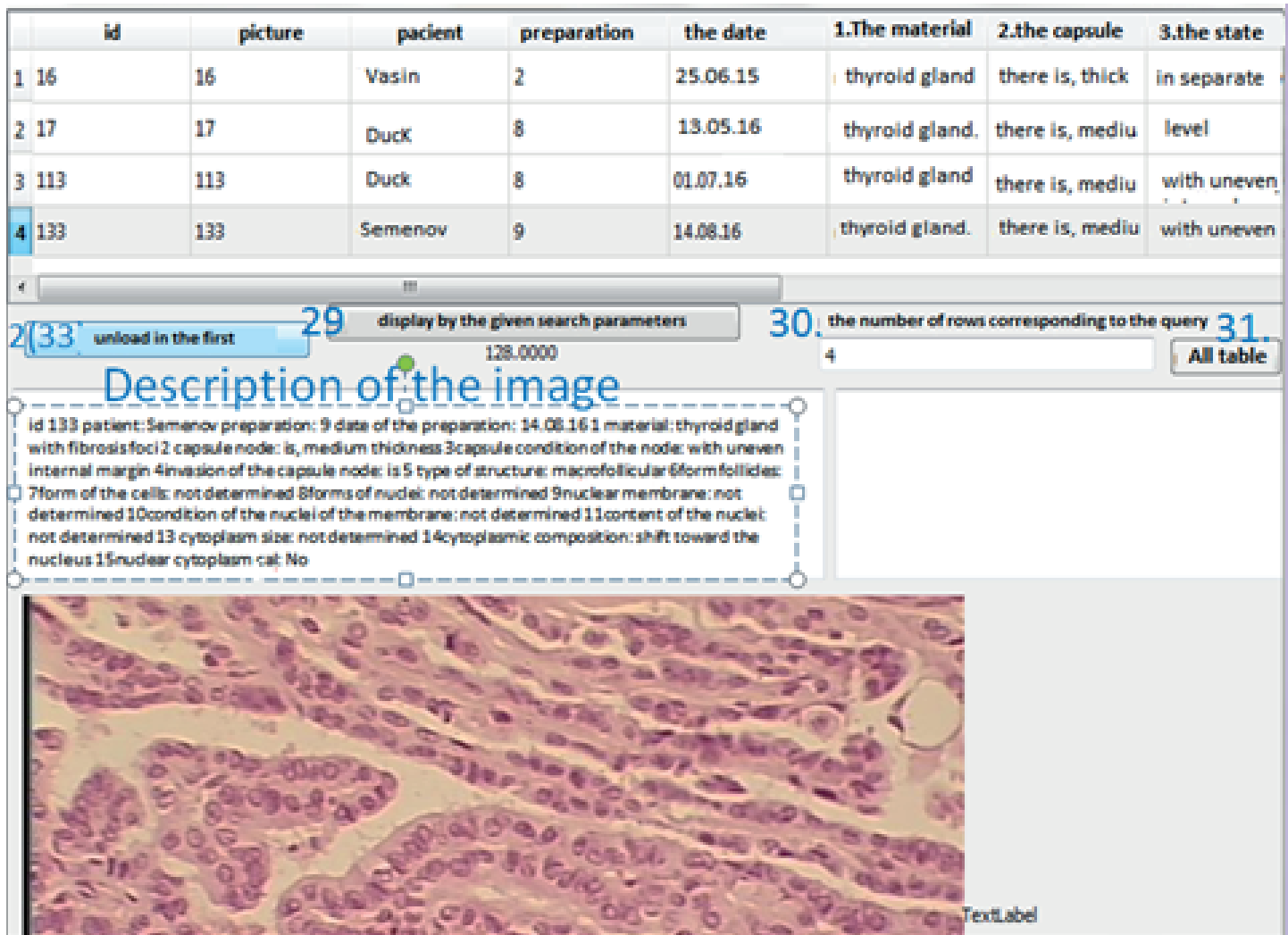

Figure 3: Search form testing of navigation and search by selected characteristics.

\section{References}

[1] V. G. Nikitaev, "Expert Systems in Information Measuring Complexes of Oncological Diagnoses" Measurement Techniques, vol. 58, no. 6, pp. 719-723, 2015.

[2] V. G. Nikitaev, "Modern measurement principles in intellectual systems for a histological diagnosis of oncological illnesses" Measurement Techniques, vol. 58, no. 4, pp. 467-470, 2015.

[3] V. G. Nikitaev, "Medical and biological measurements: Experimental hightechnology information-measuring complexes of cancer diagnosis: Problems and key points of the construction methodology" Measurement Techniques, vol. 58, no. 2, pp. 214-218, 2015.

[4] V. G. Nikitaev, "Methods and means of diagnostics of oncological diseases on the basis of pattern recognition: Intelligent morphological systems - Problems and solutions" Journal of Physics: Conference Series, vol. 798, no. 1, p. 012131, 2017.

[5] M. I. Davydov, V. Y. Selçuk, V. G. Nikitaev, O. V. Nagornov, A. N. Pronichev, V. V. Dmitriev, E. V. Polyakov, A. O. Rasulov, V. P., Kononets, S. A. Melikhov, I. S. Akimov, Z. M. Yunakov, I. V. Kardashev, A. A. Lavrova, V. K. Golovanov, A. A., Pasnik and V. E. Strigin, "Physical research methods in expert systems of oncological disease 
diagnostics" Bulletin of the Lebedev Physics Institute, vol. 42, no. 8, pp 237-239, 2015 .

[6] S.M. Zaytsev et al, A method of data structuring in the decision making support system in oncological diagnostics of prostate diseases, Journal of Physics: Conference Series, 798(1), 012132, (2017).

[7] J.T. Kwak et al, Automated prostate tissue referencing for cancer detection and diagnosis, BMC Bioinformatics, 17(1), 227, (2016)

[8] M.V. Kovylina, E. A. Prilepskaya, A. V. Govorov, V. V. D'iakov, K. B. Kolontarev, A. O. Vasilyev, A.V. Sidorenkov, P.I. Rasner, A.V. Glotov, D. Yu. Pushkar', V. G. Nikitaev and A. N. Pronichev, "Benign mimics of prostatic adenocarcinoma", Urologiia (Moscow, Russia : 1999), vol. 6, pp. 51-56, 2014.

[9] E. A. Prilepskaya, M.V. Kovylina, A. V. Govorov, A. V. Glotov, A. O. Vasilyev, K. B. Kolontarev, V. G. Nikitaev, A. N. Pronichev and D. Yu. Pushkar, "Possibilities of automated image analysis in pathology", Arkhiv Patologii, vol. 78, no. 1, pp. 51-55, 2016.

[10] S. M. Zaytsev, V.G. Nikitaev, A.N. Pronichev, B.N. Onykiy, E.V. Polyakov, A.A. Kurdin, D.Y. Pushkar, E.A. Prilepskaya, M.V. Kovilina, A.V. Govorov, A.V. Glotov, A.O. Vasilyev and K.V. Kolontarev "Computer system for remote consultations in the diagnosis of urological malignancies", Journal of Physics: Conference Series, vol. 798, no. 1, p. 012133, 2017.

[11] S. M. Zaytsev, V.G. Nikitaev, A.N. Pronichev, O.V. Nagornov, E.V. Polyakov, N.A. Romanov, D.Y. Pushkar, E.A. Prilepskaya, M.V. Kovilina, A.V. Govorov, A.V. Glotov, A.O. Vasilyev and K.V.Kolontarev "A method of data structuring in the decisionmaking support system in oncological diagnostics of prostate diseases", Journal of Physics: Conference Series, vol. 798, no. 1, p. 012132, 2017. 